\title{
Simulations and Analysis of an Infrared Prism Spectrometer for Ultra-short Bunch Length Diagnostics at the Linac Coherent Light Source
}

\author{
Julie Cass \\ Office of Science, Science Undergraduate Laboratory Internship Program
}

University of Notre Dame

SLAC National Accelerator Laboratory

Menlo Park, California

August 19, 2011

Prepared in partial fulfillment of the requirement of the Office of Science, Department of Energy's Science Undergraduate Laboratory Internship under the direction of Josef Frisch in the ICD Accelerator Physics \& Engineering Department at SLAC National Accelerator Laboratory.

Participant:

Signature

Research Advisor:

$$
\text { Signature }
$$




\begin{abstract}
Simulations and Analysis of an Infrared Prism Spectrometer for Ultra-short Bunch Length Diagnostics at the Linac Coherent Light Source.

Julie Cass (University of Notre Dame, Notre Dame, IN 46556), Josef Frisch (ICD Accelerator Physics \& Engineering, SLAC National Accelerator Laboratory, Stanford, CA 94025).

We require a means of determining the minimum achievable bunch length at the Linac Coherent Light Source (LCLS) at SLAC National Accelerator Laboratory. Current methods of measurement in the time domain do not have the resolution to measure ultra-short bunch lengths below $10 \mathrm{fs}$. An alternative method has been designed, using a single-shot near- to mid-infrared prism spectrometer for bunch length diagnosis in the frequency domain. This design requires the precision alignment of sensitive optical equipment and complete understanding of the spectrum reaching the spectrometer's detector surface. This paper provides information on preliminary simulations designed to model beam behavior in the optical set-up and at the detector surface for comparison to measured values as an indication of the current achievable optical alignment and the study of optimal detector orientation. The results of these simulations indicate the necessity for a method of greater precision in optical alignment and further study of achievable spectrometer range.
\end{abstract}




\section{INTRODUCTION}

The Linac Coherent Light Source (LCLS) at SLAC National Accelerator Laboratory is a groundbreaking free electron laser (FEL), using magnetic fields to oscillate high-energy electrons and generate ultrafast X-ray pulses [1]. This FEL is capable of producing pulses with ultra-short bunch lengths, enabling scientists to image molecules and atoms in motion, pushing the limits of technology in pursuit of a deeper understanding of fundamental chemical, biological, and physical properties of materials [2]. The interpretation and understanding of the data collected in these experiments relies on a complete understanding of the parameters used in driving the FEL. While the minimum achievable bunch length at LCLS is known to have a value below $10 \mathrm{fs}$, the precise value remains unknown. Measurement of this parameter requires a mechanism capable of probing pulses shorter than $10 \mathrm{fs}$, a scale too small to be resolved by existing time domain detectors. Scientists at the LCLS have developed an alternative design implementing a broadband infrared prism spectrometer to probe for this parameter in the frequency domain.

This new approach is centered on the concept that information about the bunch length can be extracted from the spectrum of optical transition radiation generated by the electron beam. This radiation can be characterized as a relativistic effect, resulting in the emission of electromagnetic radiation as a charge particle approaches a conducting foil. The implementation of an inverse Fourier transform of the transition radiation spectrum allows for the inversion of spectral information for analysis in the time domain. This provides an absolute means for

calculation of the FEL bunch length from the information contained in the transition radiation 
spectrum. The wavelength range of interest for the transition radiation from the LCLS beam is within the near- to mid-infrared range.

Transition radiation from FEL electrons is expanded and collimated using precision optics [3] and steered to the spectrometer prism. The index of refraction of the prism material is wavelength dependent, creating a dispersion relation between the wavelengths comprising the radiation beam and the angle of refraction as the light emerges from the prism. The dispersed light is focused onto the detecting surface of a pyroelectric detector array [4]. The resulting spectrum will then be used in the inverse Fourier analysis to determine the FEL bunch length. It is crucial for the success of this approach that the spectrometer is designed with precisely aligned optics for propagation of transition radiation from the foil to the detector. For this purpose, a method for analyzing the behavior of the beam in the optical system of the spectrometer has been determined. We require a calculation of both how the beam would behave for an ideal system and for a system containing aberrations with the introduction of alignment errors.

\section{METHODS AND MATERIALS}

\section{Spectrometer Design}

The infrared spectrometer design is comprised of an electronic system to drive and collect data from the pyroelectric line detector and an optical set-up employing lenses and mirrors to expand, collimate and steer the beam towards this detector. A diagram of the optical system as it would be implemented in the LCLS is provided in Figure 1. 


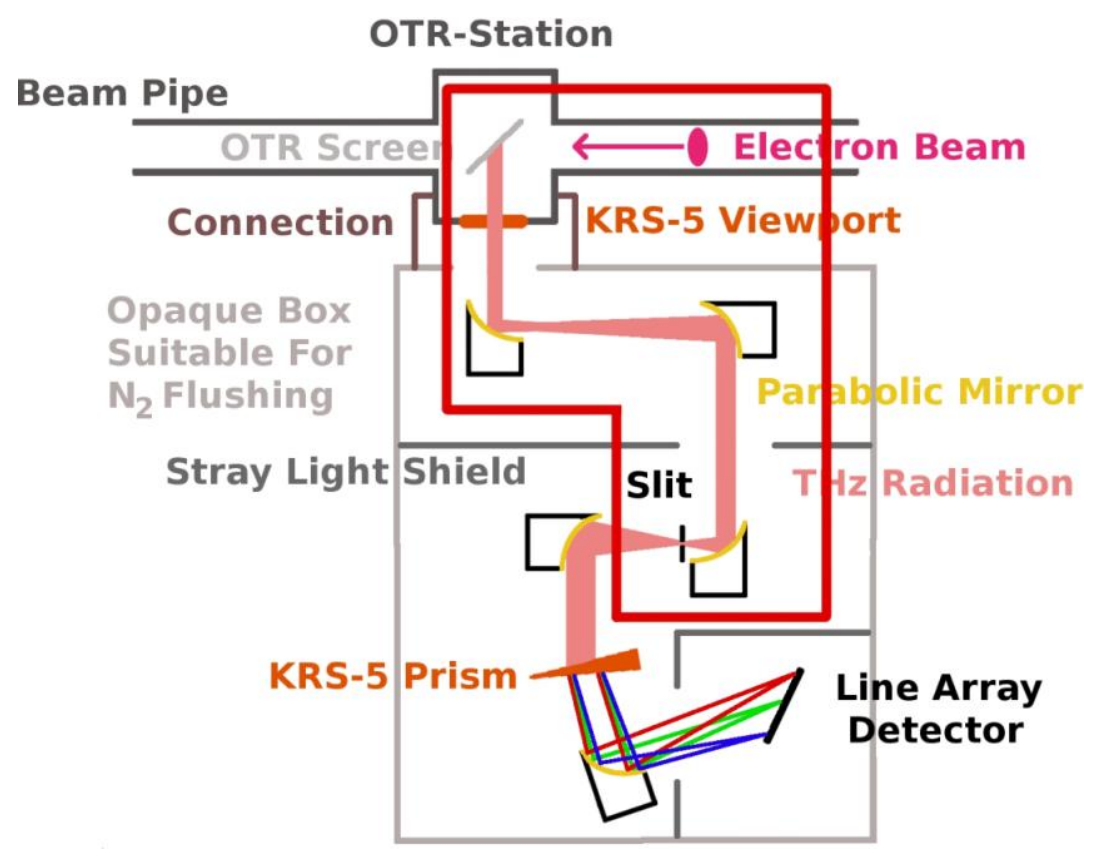

Figure 1. Preliminary optical layout for the prism spectrometer

The mirrors used in this optical design are gold-coated, 90-degree, off-axis parabolic mirrors (OAPs). Preliminary calculations for this project showed that the optical transition radiation form the LCLS is likely to fall in the infrared wavelength range. These mirrors were chosen for their reflectivity in the wavelength range of interest. They are particularly difficult to align, due to the challenge in locating the off-axis central point. A Helium-Neon (HeNe) laser emitting light in the visible range at $632.816 \mathrm{~nm}$ was initially used for positioning of the system elements and a Thallium Bromo-Idodide (KRS-5) crystal was selected to act as the spectrometer prism, dispersing the transition radiation over the detector. This material was chosen for its dispersive and transmission properties in the range of wavelengths of interest.

\section{MATLAB Simulation for Idealized Modeling}

As proper alignment is essential to our project, we required a calculation of the tolerances for beam behavior while navigating the optical system. A mathematical interpretation of the 
optical system was programmed in MATLAB [6] to graphically simulate the size of the beam as it is passed through various optical elements using "ray transfer matrix analysis" [7]. The programming software MATLAB was selected for this simulation due to its capabilities in matrix-based calculation. In the ray transfer matrix approach to optical analysis, light rays are represented as vectors in terms of height $r$, and angle of inclination $\theta$ relative to the optical axis. Each optical element or length of free space propagated by the beam is represented by $2 \times 2$ matrix; when the initial light ray is multiplied by the ray transfer matrix, the result is a new vector, containing information about the outgoing beam height and angle of inclination. Examples of ray transfer matrices for light refraction through a lens with focal length $f$ and the propagation of a ray through free space of length $d$ are provided in Equations 1 and 2 respectively.

$$
\begin{aligned}
& \left(\begin{array}{l}
r_{f} \\
\theta_{f}
\end{array}\right)=\left(\begin{array}{cc}
1 & 0 \\
-1 / f & 1
\end{array}\right)\left(\begin{array}{l}
r_{i} \\
\theta_{i}
\end{array}\right) \\
& \left(\begin{array}{l}
r_{f} \\
\theta_{f}
\end{array}\right)=\left(\begin{array}{ll}
1 & d \\
0 & 1
\end{array}\right)\left(\begin{array}{l}
r_{i} \\
\theta_{i}
\end{array}\right)
\end{aligned}
$$

If we represent each successive optical element or length of free space (at $100 \mu \mathrm{m}$ intervals) in the system as ray transfer matrix $M_{i}$, we can represent the compounded effects of the entire optical system with a single ray transfer matrix $\mathrm{M}$ :

$$
M=\prod_{i=1}^{n} M_{i}
$$

where $\mathrm{n}$ is the total number of optical elements and $100 \mu \mathrm{m}$ intervals of free space in the system. While basic ray transfer matrix analysis is designed for modeling ideal behavior of light rays with negligible waist size, our system required ray transfer analysis of Gaussian beam 
propagation, as the HeNe laser used to align the system produces a Gaussian beam. In order to incorporate the effects of diffraction into our design, we generated a vector to represent the initial Gaussian beam in terms of a complex beam parameter $q$, calculated by the following equation:

$$
\frac{1}{q}=\frac{1}{R}-\frac{D}{\pi w^{2}}
$$

where $q$ is the complex beam parameter, $R$ is the radius of curvature of the beam, $\lambda$ is the wavelength of the beam and $w$ is the beam waist size or beam diameter [8]. With this notation, the equation modeling the transformation of the initial beam to a final beam through ray transfer analysis takes on the modified form:

$$
\left(\begin{array}{c}
q_{f} \\
1
\end{array}\right)=k \cdot M \cdot\left(\begin{array}{c}
q_{i} \\
1
\end{array}\right)
$$

where the beam is now represented by a vector containing the complex beam parameter and $k$ is a constant chosen to maintain the normalization of the bottom component of the beam vector. Carrying out this multiplication and solving for the inverse $q$ value leaves us with:

$$
\frac{1}{q_{f}}=\frac{M(2,1)+M(2,2) / q_{i}}{M(1,1)+M(1,2) / q_{i}}
$$

giving us a way to find the new waist size from the definition of the beam parameter $q$ and the elements contained in the ray transfer matrices and initial beam parameter. From this information, the MATLAB code calculates the beam waist at each element and free space interval and outputs a graph of these waist sizes.

This simulation was designed with matrices representing ideal versions of each optical element; off-axis parabolic mirrors and lenses are treated according to the thin lens approximation. The errors in alignment are not accounted for; the program offers an outline for 
behavior in a system with infinite precision as a guideline for proper alignment and a means of comparison between ideal beam behavior and the behavior, or beam sizes, seen in the actual setup.

\section{ZEMAX Simulation}

Disagreements between the measured beam sizes and those calculated in the MATLAB simulation were anticipated due to limitations in the precision aligning of optics. We wished to determine the spectrometer tolerances achievable considering these limitations. For a more accurate representation of the design and calculation of this resolution, the optical simulation software ZEMAX was used [9]. This software provides the ability to trace rays of light as they are reflected, refracted, and dispersed in an optical system.

The spot size was analyzed at two crucial points in the optical system: the focal points of the first and third OAPs, with the latter corresponding to the proposed location of the line array detector. As these are the two tightest focuses in the optical design, they are excellent test points for the design tolerances in angular misalignments. The beam sizes measured and simulated in this project are the $1 / \mathrm{e}^{2}$ beam diameters. For analysis of the beam size at the focal point of the first OAP, a ZEMAX simulation of a single 90-degree OAP with a $50.8 \mathrm{~mm}$ focal length and mirror diameter perpendicular to the optical axis of $50.8 \mathrm{~mm}$ was generated. The $3 \mathrm{D}$ plot of this mirror produced by ZEMAX is provided in Figure 2. 


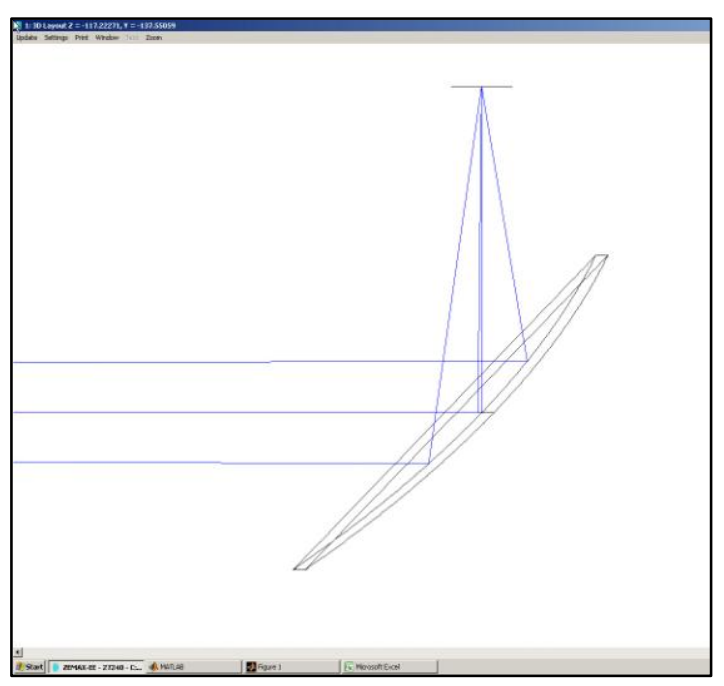

Figure 2. ZEMAX ray tracing of reflection off of a 90-degree off-axis parabolic mirror. Light rays are incident from the left side of the image and are focused by the OAP.

As the HeNe laser used has a beam output diameter $2 w_{0}$ of $2 \mathrm{~mm}$ and is expanded in a pair of lenses, or a telescope, to approximately 17 times its initial size, the entrance beam size for the simulation was set to $33 \mathrm{~mm}$. The beam size at the OAP focus was first calculated for the case of a perfectly aligned mirror. Mirror tilts on the order of a tenth of a degree about the horizontal, vertical and optical axes were added in keeping with the estimated tolerances for physical alignment of the optics. These angles were adjusted until a beam size similar to the beam size measured on the optical table was reached. The tilts needed to cause these beam sizes were recorded for comparison with estimated order of misalignment error.

The design for testing beam sizes due to aberrations at the final detector location was more complex. While the functionality of the spectrometer depends on the dispersion of wavelengths through the prism, this dispersion also introduces chromatic aberrations in the focal plane of the spectrum. Previous simulations of the beam path indicate that, due to these chromatic aberrations, the wavelength-dependent focal plane is not perpendicular to the optical axis; a 44 degree tilt of the detector plane was required to minimize these effects [10]. We 
wished to create a new simulation of this portion of the spectrometer design to account for differences in mirror diameters and beam sizes, as well as refocusing the system to the best focus for all wavelengths of dispersed light. From this model we could then test the angular tolerances and determine the new optimal detector tilt.

The simulation was first designed to focus several wavelengths of light at the detector to simulate actual conditions for application in LCLS. While chromatic aberrations make perfect foci impossible to produce, we could optimize the optical system in ZEMAX to achieve minimum beam diameters and sharpest possible foci. A simple example of the process is shown for a basic plano-concave lens in Figure 3a-b. When the ZEMAX function 3D-plot is implemented for a simple lens design (Figure 3a) we can see the unclear focus produced as different rays of light focus at different points.
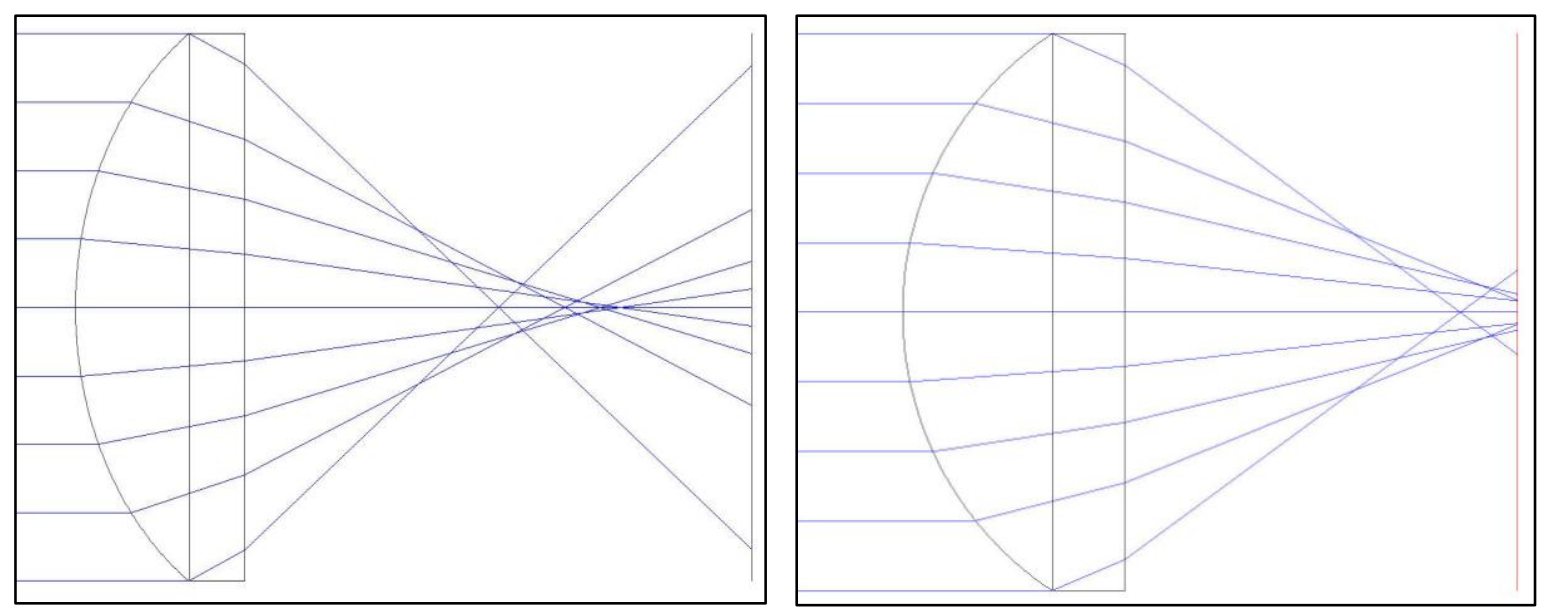

Figure 3a and 3b. ZEMAX tracing of light rays intiated at the far left of the image, are refracted through plano-concave lens with spherical aberrations onto an image plane (line at far right), shown before and after focusing optimization

Owing to the chromatic aberrations, the "focal length" specified for the lens is no longer the point of smallest beam radius. Instead, we defined a new adjusted focal length as the point where the tightest beam waist is located. Through use of the Merit Function Editor in ZEMAX, system 
parameters specified by the user in the Lens Data Editor as variables may be adjusted, allowing for system optimization. The Geometric Encircle Energy (GENC) merit function was used for the focusing of elements in this simulation. GENC places the image plane closest to the point at which a user-specified fraction of the beam energy falls within a user-specified beam radius, constraining beam size at the image plane. Using the Optimization feature of ZEMAX allows the software to adjust any "variable" parameters in the lens data editor, such as distance from the object to the image plane, in order to meet the target encircled energy values as closely as possible. Figure $3 \mathrm{~b}$ displays the resulting 3D plot when the GENC merit function was used to constrain the greatest possible amount of beam energy to the smallest possible radius at the image plane. This technique for image focus, once understood for simple cases as the lens shown above, was then applied to our optical design for precision focusing of OAPs about the prism and detector for a measurement of the new detector tilt needed and angular tolerances in beam alignment. The GENC target fraction of beam energy set in order to simulate the $1 / \mathrm{e}^{2}$ beam diameter for comparison with the measured beam sizes.

With a design completed for the spectrometer dispersion of several wavelengths of light, the model was adjusted to simulate only the beam path of the HeNe laser and the system was reoptimized for this single wavelength. A ZEMAX plot of this model is given in Figure 4. 


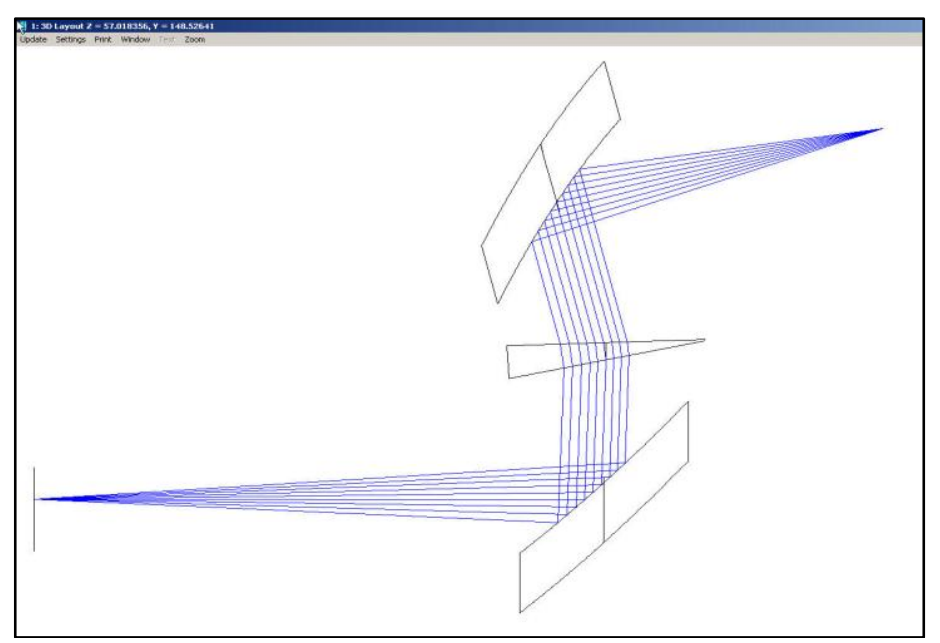

Figure 4. ZEMAX plot of OAP focusing of HeNe light onto the detector plane (line on the far left) after traveling through the final two OAPs and prism

To determine the angular adjustments needed to cause a beam size similar to the measured beam size, coordinate breaks with angular tilts were added to the design. Angular tilts about the optical, vertical, and horizontal axes were added and the system was optimized for each new design angle. The tilts were adjusted until the simulated beam size was similar to the measured beam size and the resulting tilts were compared to the expected magnitude of tilt misalignments. We could then consider whether the aberrations in measured beam sizes were in fact due to misalignments and whether they were close enough to the values predicted by MATLAB and ZEMAX to be negligible in spectrometer functionality.

\section{RESULTS}

Figure 5 below displays a plot of the beam waist results produced through the implementation of the MATLAB optical simulation code. 


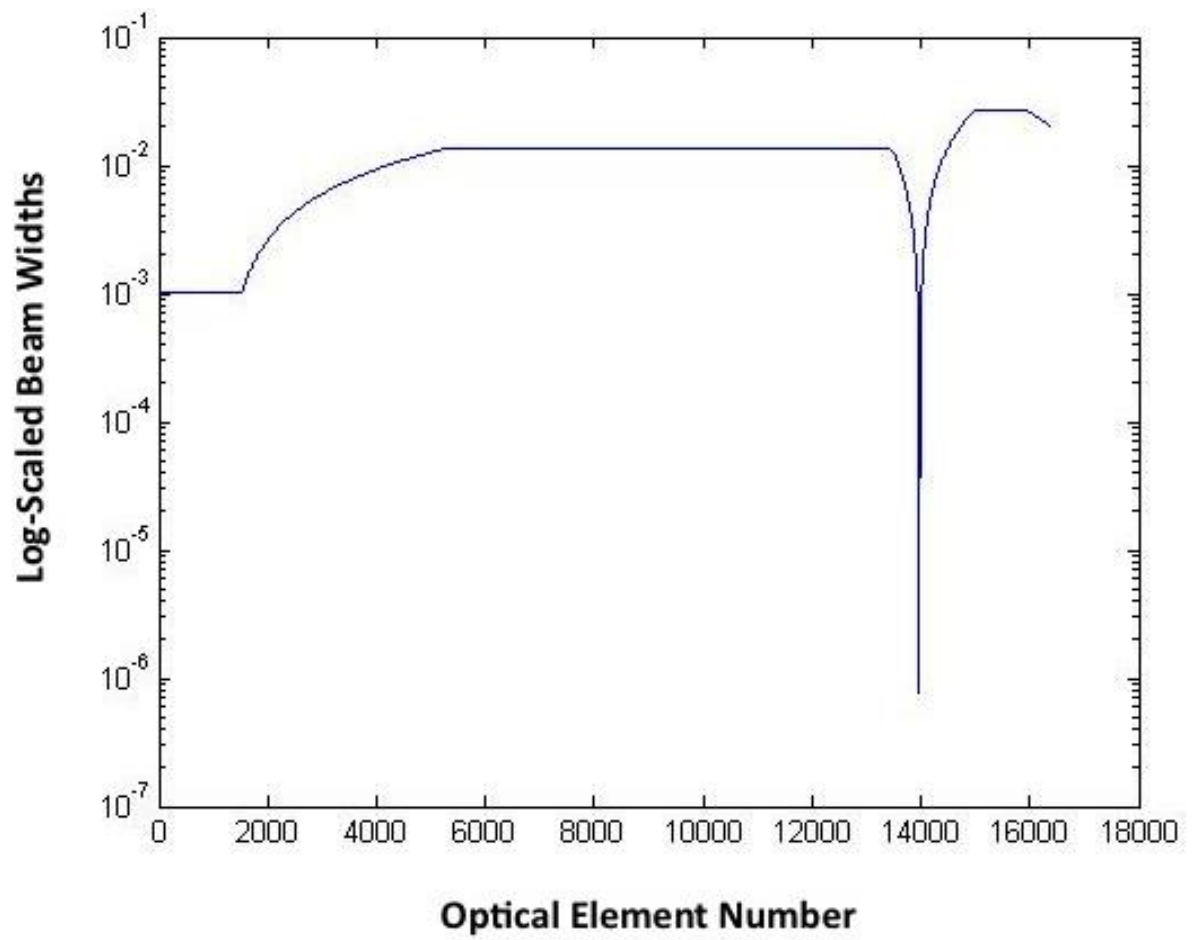

Figure 5. Log-scaled plot of beam size at various points in the spectrometer optics predicted by MATLAB simulation

The Optical Element Numbers given in the x-axis represent which step through the optical system each element falls at. The step size for this system was $0.1 \mathrm{~mm}$. Each Optical Element is either a distance step of this size or an optical element itself.

At the first focal point of interest, following light reflection from the first parabolic mirror, we measured a beam diameter of approximately $172 \mu \mathrm{m}$. Our simulations in MATLAB predict a beam diameter of approximately $65 \mu \mathrm{m}$. To determine whether the magnification of the measured beam size by three times the predicted size could be the result of misalignments, we compared these numbers to those generated by the ZEMAX simulation. The Spot Diagram display in ZEMAX providing the beam diameter at this focal point through general ray tracing predicted a beam size of $0 \mu \mathrm{m}$ without any mirror tilts or aberrations. Tilts of approximately 0.4 degrees both along the optical axis and perpendicular to the optical table were needed in order to produce a beam with diameter equal to the measured beam diameter. 
At the focal point of the third parabolic mirror, with no detector tilt implemented, we measured the beam diameter of approximately $280 \mu \mathrm{m}$. MATLAB simulations predict a beam size of approximately $37.5 \mu \mathrm{m}$ at this location. It was determined that the disagreements between these two values were also likely the result of limits in precision alignment of the system, though these effects seemed much greater at the detector location than at the focal point of the first parabolic mirror. This hypothesis was tested with the study of beam sizes predicted by the ZEMAX model. Without aberrations added, this simulation predicted a beam diameter of $14 \mu \mathrm{m}$. Tilts of approximately 1 degree both about the vertical, horizontal, and optical axes were needed to cause beam sizes comparable to the measured beam size. A table containing all relevant measured and simulated beam sizes is provided in Table 1.

\begin{tabular}{|c|c|c|c|c|}
\hline & Measured & MATLAB & ZEMAX & $\begin{array}{c}\text { ZEMAX Tilts } \\
\text { Required }\end{array}$ \\
\hline $\begin{array}{c}\text { 50.8 mm OAP } \\
\text { Focal Point }\end{array}$ & $172 \mu \mathrm{m}$ & $65 \mu \mathrm{m}$ & $0 \mu \mathrm{m}$ & $\mathbf{0 . 4}^{\circ}$ \\
\hline $\begin{array}{c}\text { Detector } \\
\text { Surface }\end{array}$ & $280 \mu \mathrm{m}$ & $37.5 \mu \mathrm{m}$ & $14 \mu \mathrm{m}$ & $1^{\circ}$ \\
\hline
\end{tabular}

Table 1. Table of measured and simulated beam diameters $(\mu \mathrm{m})$ at two points of interest in the optical system, and simulation tilts needed to produce the measured beam sizes

With a new simulation designed to match the design parameters of our spectrometer, multiple wavelengths of light were added to the simulation and the detector tilt and distance were optimized for focusing of all wavelengths. A new detector tilt of 45 degrees was determined to minimize chromatic aberrations at the detector plane. While this tilt provided a decent focus for all wavelengths of light, this design created a dispersion of approximately $16 \mathrm{~mm}$. The detector, however, has a length of $14 \mathrm{~mm}$ and not all wavelengths of light transmitted by the KRS-5 prism would fit on the detector for analysis. Ray tracing images of this portion of the system and light 
focusing at the detector are given in Figure 6. A spot diagram of all wavelengths falling on the detector surface is given in Figure 7 and a table listing the wavelengths plotted in these simulations is given in Table 2.
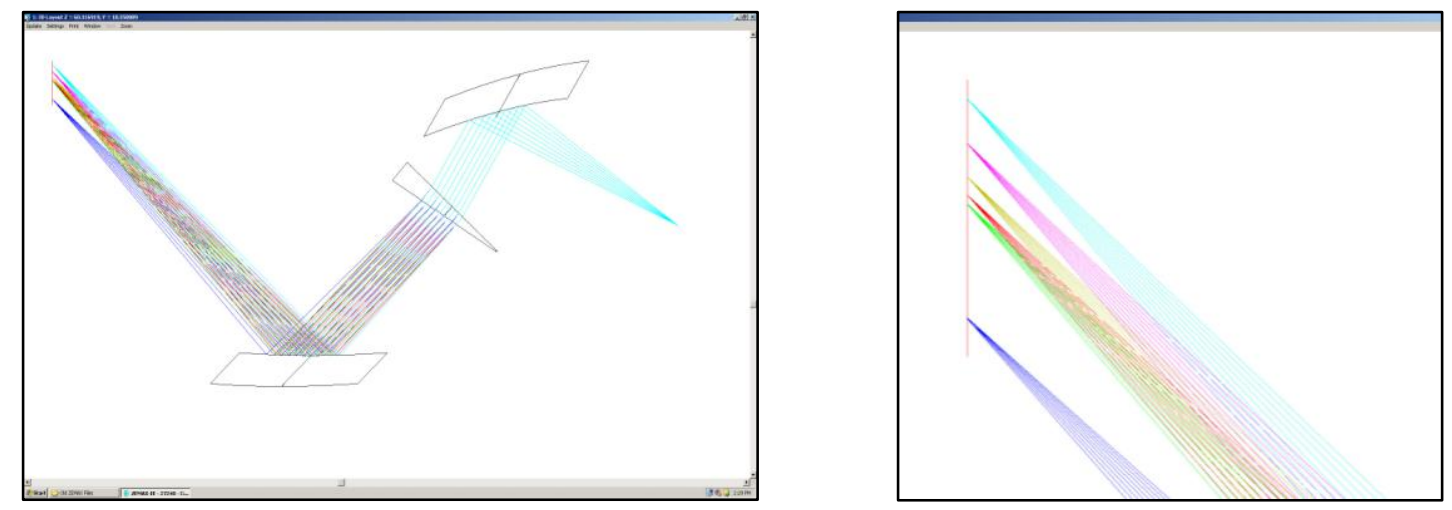

Figure 6. Ray tracing for focusing of several wavelengths onto the detector plane. Left: The simulated beam initiates at the far right, is reflected by the $105.6 \mathrm{~mm}$ OAP, disperses through the prism, reflects off of the $177.8 \mathrm{~mm}$ OAP and falls on the detector surface. Right: Close-up of all wavelengths focusing onto the detector plane.

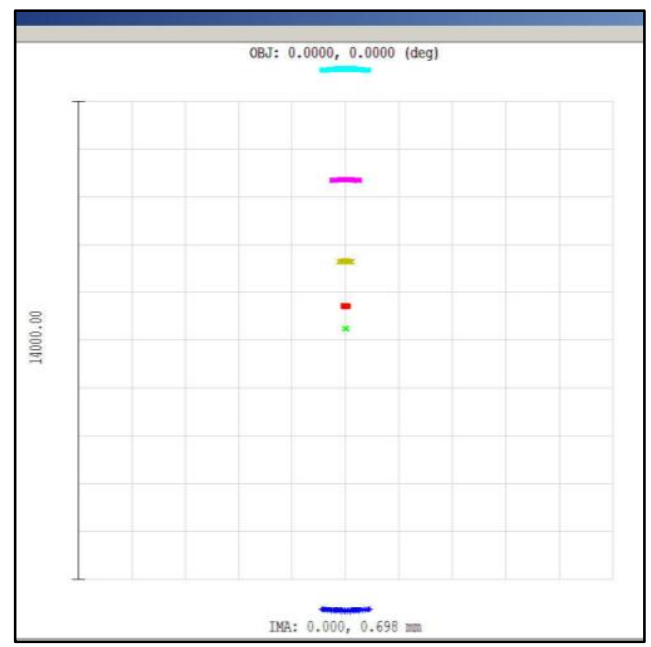

Figure 7. Spot diagram for the detector surface. The vertical axis represents the length of the detector array. Each wavelength is confined to a thin strip along the vertical or detector axis 


\begin{tabular}{|c|c|}
\hline \multicolumn{2}{|c|}{ Wavelengths $(\mu \mathrm{m})$} \\
\hline Blue & $\mathbf{0 . 6 3 2}$ \\
\hline Green & 3.39 \\
\hline Red & 10.6 \\
\hline Gold & $\mathbf{2 0 . 0}$ \\
\hline Magenta & 30.0 \\
\hline Turquoise & 39.0 \\
\hline
\end{tabular}

Table 2. List of wavelengths $(\mu \mathrm{m})$ plotted in the ZEMAX simulation shown in Figure 7.

\section{DISCUSSION AND CONCLUSION}

Comparisons of the measured beam size and MATLAB simulated beam sizes at both the focal point of the 50.8mm OAP and the detector plane yielded measured results larger than those simulated by MATLAB. This result was consistent with our hypothesis that discrepancies in these beam sizes could be the result of minor errors in alignment. When the system was modeled in ZEMAX and tilts were added to simulate mirror misalignment, we found that the tilts necessary to match the measured beam sizes were less than or equal to one degree. We believe that precision in mirror alignment is limited to tolerances greater than one degree. However, the aberrations found in our system are great enough, that we believe a superior method for precision alignment is needed for the spectrometer to function properly. We will require further research and testing to develop a method for fine-tuning this alignment for a closer match between the predicted and measured beam sizes.

Spectrometer design considerations originally predicted the transmission range of KRS-5 prism to be the limiting factor in the range of wavelengths detected in the spectrometer. Through our ZEMAX simulations, we found that no detector tilt can be implemented in our current design to allow all wavelengths transmitted by this prism. This result implies that either our 
spectrometer detection range will be bounded for larger wavelengths at $38 \mu \mathrm{m}$ rather than $40 \mu \mathrm{m}$ or the design will need to be changed. We believe that this decrease in detector range is small enough that the current spectrometer design will be sufficient. The spectrometer contains aberrations large enough that a new method for alignment is needed but has a detection range sufficient to produce the spectrum needed for FEL bunch length diagnosis.

\section{ACKNOWLEDGEMENTS}

This research was carried out at the SLAC National Accelerator Laboratory between May 23 and August 19, 2011 under the direction of Josef Frisch. The author would like to thank the United States Department of Energy and the SLAC National Accelerator Laboratory for supporting this research through the creation, organization, and funding of the Summer Undergraduate Laboratory Internship (SULI) program. She would also like to thank her mentor Joe Frisch and research partners Gilles Dongmo-Momo and Kiel Williams. Thanks also go to Alan Fisher for his guidance in optical simulation design. 


\section{REFERENCES}

[1] Y. Ding et al., "Measurements and Simulations of Ultralow Emittance and Ultrashort Electron Beams in the Linas Coherent Light Source", PRL 102, 254801, 2009

[2] Linear Coherent Light Source http://lcls.slac.stanford.edu/

[3] K. Williams, "Optical Design of a Broadband Infrared Spectrometer for Bunch Length Measurement at the Linac Coherent Light Source," SLAC National Accelerator Laboratory SULI Program 2011, Palo Alto, CA

[4] Pyreos Ltd, http://www.pyreos.com/

[5] G. Dongmo-Momo, "Infrared Spectroscope for Electron Bunch-length Measurement: Heat Sensor Parameters Analysis," SLAC National Accelerator Laboratory SULI Program, Palo Alto, CA 2011

[6] MATLAB 7.0.4, The MathWorks Inc.

[7] Bahaa E. A. Saleh and Malvin Carl Teich (1991). Fundamentals of Photonics. New York: John Wiley \& Sons. Section 1.4, pp. 26-36

[8] Gaussian Beams http://www.rp-photonics.com/

[9] Radiant ZEMAX LLC, http://www.zemax.com

[10] C. Behrens et al., "Design of a Single-Shot Prism Spectrometer in the Near- and MidInfrared Wavelength Range for Ultra-Short Bunch Length Diagnostics”, DIPAC'11, Hamburg, Germany, 201 